\title{
Moving contact line on chemically patterned surfaces
}

\author{
XIAO-PING WANG ${ }^{1}$, TIEZHENG QIAN ${ }^{1}$ \\ AND PING SHENG ${ }^{2}$ \\ ${ }^{1}$ Department of Mathematics, The Hong Kong University of Science and Technology, Clear Water Bay, \\ Kowloon, Hong Kong, China \\ ${ }^{2}$ Department of Physics and Institute of Nano Science and Technology, The Hong Kong University \\ of Science and Technology, Clear Water Bay, Kowloon, Hong Kong, China
}

(Received 20 November 2007 and in revised form 29 February 2008)

We simulate the moving contact line in two-dimensional chemically patterned channels using a diffuse-interface model with the generalized Navier boundary condition. The motion of the fluid-fluid interface in confined immiscible two-phase flows is modulated by the chemical pattern on the top and bottom surfaces, leading to a stick-slip behaviour of the contact line. The extra dissipation induced by this oscillatory contact-line motion is significant and increases rapidly with the wettability contrast of the pattern. A critical value of the wettability contrast is identified above which the effect of diffusion becomes important, leading to the interesting behaviour of fluid-fluid interface breaking, with the transport of the non-wetting fluid being assisted and mediated by rapid diffusion through the wetting fluid. Near the critical value, the time-averaged extra dissipation scales as $U$, the displacement velocity. By decreasing the period of the pattern, we show the solid surface to be characterized by an effective contact angle whose value depends on the material characteristics and composition of the patterned surfaces.

\section{Introduction}

The question of how immiscible two-phase fluids flow over rough surfaces or chemically patterned surfaces has long been of interest to academic and industrial communities. In order to exploit the potential utility of microfluidic devices, it is important to develop a fundamental understanding of the behaviour for fluids flowing over patterned surfaces. Modelling of immiscible two-phase flows over solid surface has eluded accurate continuum description for many decades. At the heart of this problem is the boundary condition for the moving contact line (MCL), where the fluid-fluid interface intersects the solid wall (Huh \& Scriven 1971; Dussan V. \& Davis 1974; Dussan V. 1979; de Gennes 1985). It has been shown by Dussan V. \& Davis (1974) that under usual hydrodynamic assumptions, namely incompressible Newtonian fluids, the no-slip boundary condition, and smooth rigid solid walls, the velocity field is multi-valued at the MCL, and the tangential force exerted by the fluids on the wall in the vicinity of the MCL is unbounded, implying an infinite rate of viscous dissipation. Numerous models have been proposed over the years, aiming to resolve this classical hydrodynamic problem. An incomplete list includes the kinetic adsorption/desorption model by Blake \& Haynes (1969), the slip models by Hocking (1977), Huh \& Mason (1977), and Zhou \& Sheng (1990), and the diffuse-interface 
models by Seppecher (1996), Jacqmin (2000), Chen, Jasnow \& Vinals (2000), Pismen $\&$ Pomeau (2000) and Briant \& Yeomans (2004). It is interesting to note that, while the divergence of viscous dissipation can be removed by allowing some slip in the vicinity of the MCL, the diffuse-interface models provide a different resolution. That is, an effective interfacial slip can be maintained by diffusive transport even if the no-slip boundary condition is still satisfied.

The recent discovery of the generalized Navier boundary condition (GNBC) by Qian, Wang \& Sheng (2003) has resolved the MCL conundrum for immiscible twophase flows over homogeneous surfaces. The GNBC states that the slip velocity is proportional to the total tangential stress - the sum of the viscous stress and the uncompensated Young stress; the latter arises from the deviation of the fluid-fluid interface from its static configuration. By combining the GNBC with the CahnHilliard $(\mathrm{CH})$ diffuse-interface model (Cahn \& Hilliard 1958), we have obtained a continuum description for the contact line hydrodynamics in immiscible twophase flows. Our continuum calculations based on the new slip boundary condition quantitatively reproduce the molecular dynamics (MD) simulation results (Qian, Wang \& Sheng 2003, 2004). It is worth emphasizing that, by using a diffuseinterface model, the stress singularity can be removed by either diffusion or slip, or both, depending on the physical system under investigation. For the fluid systems in our MD simulations, the slip is the dominant mechanism. More recently, we have presented a variational derivation of the GNBC through the principle of minimum energy dissipation (Qian, Wang \& Sheng 2006), formulated by Onsager for small perturbations away from equilibrium (Onsager 1931a,b; Onsager \& Machlup 1953). This derivation reveals that the slip boundary condition at the fluid-solid interface is consistent with the general principle of irreversible thermodynamic processes.

Numerous studies have shown that the behaviour of a binary mixture is significantly affected by the wetting properties of the underlying surface pattern. Consider a surface patterned with hydrophilic and hydrophobic domains in contact with a liquid (e.g. water); the corresponding interface will have a position-dependent free energy that reflects the underlying surface pattern. The liquid wets the hydrophilic domains but dewets the hydrophobic domains. As a result, the surface pattern will modulate the shape of the liquid layer. The morphology of the liquid microstructures depends sensitively on parameters such as the dimensions and contact angles of the pattern and may undergo morphological transition between different states (Gau et al. 1999; Darhuber et al. 2000). The dynamics of two-phase fluids or drops is even more significantly affected by the geometric and physical properties of the chemically patterned surfaces. An experimental investigation was performed by Cubaud \& Fermigier (2004) on advancing contact lines of large drops spreading on chemically patterned surfaces with different surface geometries. Experiments and numerical simulations were performed by Kusumaatmaja et al. (2006) exploring the behaviour of liquid drops moving across a surface patterned with hydrophobic and hydrophilic stripes. It was observed that the period of the pattern is reflected in the time variation of the drop velocity as well as drop shape deviation (from spherical). Simulations by Kuksenok et al. (2003) have shown that when a phase-separated binary fluid is driven to flow past chemically patterned surfaces in a microchannel, the fluid exhibits unique morphological instabilities characterized by periodic formation of monodisperse droplets. We note that most of the continuum simulations for dynamics of two-phase fluids on chemically patterned surfaces have been based on models with no-slip boundary conditions. 
In this paper, we extend the GNBC-based continuum calculations to twodimensional chemically patterned channels, and present a numerical study of the effects of the surface pattern on the MCL and moving interface. In particular, we are interested in how the motion of the contact line and interface is determined by the interplay between the driving velocity and the chemical (contact angles) and geometrical characteristics of the patterned surfaces. Our simulations show that oscillatory (stick-slip) motion of the fluid-fluid interface occurs on the chemical patterned surface. The extra dissipation induced by this oscillatory contact-line motion is due to the capillary waves generated at the fluid-fluid interface (Zhou \& Sheng 1990) and has certain universal features. The scaling of the extra dissipation (when time averaged) with the displacement velocity $U$ is found to be $\sim U$. We also show that by decreasing the period of the pattern, the solid surface can be characterized by an effective contact angle whose value depends on the material characteristics and composition of the patterned surface. If the wettability contrast of the patterned surface is strong enough, the effect of diffusion becomes important, leading to the interesting behaviour of fluid-fluid interface breaking, with the transport of the nonwetting fluid being assisted and mediated by rapid diffusion through the wetting fluid. As a consequence, a droplet appears to 'jump' across the non-wetting section of the pattern through diffusion.

The paper is organized as follows. In $\$ 2$ we describe the diffuse-interface model, the generalized Navier boundary condition (GNBC) and their numerical implementation on chemically patterned surfaces. Numerical results and a discussion are presented in $\S 3$. The paper concludes with a few remarks in $\S 4$.

\section{The diffuse-interface model}

\subsection{The Cahn-Hilliard Navier-Stokes equations with the GNBC}

To describe the continuum model for contact-line motion, we start from the $\mathrm{CH}$ free energy functional (Cahn \& Hilliard 1958):

$$
F[\phi(\boldsymbol{r})]=\int \mathrm{d} \boldsymbol{r}\left[\frac{K}{2}(\nabla \phi)^{2}+f(\phi)\right],
$$

where $\phi(\boldsymbol{r})$ is the phase field measuring the local composition (relative concentration) of the two-phase fluid, and $f(\phi)$ is a function of the local composition, given by $f(\phi)=-r \phi^{2} / 2+u \phi^{4} / 4$ which has a double-well structure for modelling the fluid components' immiscibility. A diffuse interface between the two equilibrium phases $\phi_{ \pm}= \pm \sqrt{r / u}$ (at which $\mathrm{d} f / \mathrm{d} \phi=0$ and $\mathrm{d}^{2} f / \mathrm{d} \phi^{2}>0$ ) can be stabilized by the $\mathrm{CH}$ free energy functional. Here the parameters $K, r$, and $u$ can be determined from the two equilibrium phases $\phi_{ \pm}= \pm \sqrt{r / u}(= \pm 1$ here $)$, the interfacial tension $\gamma=2 \sqrt{2 K r} \phi_{ \pm}^{2} / 3$, and the characteristic length scale $\xi=\sqrt{K / r}$ measurable in the static interfacial profile (the interfacial thickness is of the same order of magnitude as $\xi$ ). The two coupled equations of motion are the convection-diffusion equation for the phase field and the Navier-Stokes equation in the presence of the capillary force density:

$$
\begin{gathered}
\frac{\partial \phi}{\partial t}+\boldsymbol{v} \cdot \nabla \phi=M \nabla^{2} \mu \\
\rho\left[\frac{\partial \boldsymbol{v}}{\partial t}+(\boldsymbol{v} \cdot \nabla) \boldsymbol{v}\right]=-\nabla p+\nabla \cdot \boldsymbol{\sigma}^{v}+\mu \nabla \phi+\boldsymbol{f}_{e},
\end{gathered}
$$


together with the incompressibility condition $\nabla \cdot v=0$. Here $\nabla^{2} \equiv \nabla \cdot \nabla$ denotes the Laplace operator, $M$ is the mobility coefficient, $\mu=-K \nabla^{2} \phi-r \phi+u \phi^{3}$ is the chemical potential defined from the $\mathrm{CH}$ free energy functional $F$ according to $\mu=\delta F / \delta \phi$, $\rho$ is the mass density of the fluid, $p$ is the pressure, $\boldsymbol{\sigma}^{v}=\eta\left[(\nabla \boldsymbol{v})+(\nabla \boldsymbol{v})^{T}\right]$ is the Newtonian viscous stress tensor with $\eta$ being the viscosity, $\mu \nabla \phi$ is the capillary force density, and $f_{e}$ is the external force. The boundary conditions at the solid surface are the impermeability condition $\partial_{n} \mu=0$ (which requires the normal $(n)$ component of the diffusive current $\boldsymbol{J}=-M \nabla \mu$ to vanish at the solid surface) and $v_{n}=0$, the relaxational equation for $\phi$ at the solid surface:

$$
\frac{\partial \phi}{\partial t}+v_{\tau} \partial_{\tau} \phi=-\Gamma L(\phi)
$$

and the slip boundary condition governing the tangential momentum transport across the fluid-solid interface:

$$
\beta(\phi) v_{\tau}^{s l i p}=-\eta\left(\partial_{n} v_{\tau}+\partial_{\tau} v_{n}\right)+L(\phi) \partial_{\tau} \phi
$$

Here $\tau$ denotes the direction tangent to the solid surface (for two-dimensional flows), $n$ denotes the outward surface normal, $\Gamma$ is a positive phenomenological parameter, $L(\phi)=K \partial_{n} \phi+\partial \gamma_{f s}(\phi) / \partial \phi$ in which $\gamma_{f s}(\phi)$ is a function of the local composition at the solid surface measuring the fluid-solid interfacial free energy per unit area, $\beta(\phi)$ is the slip coefficient which may depend on the local composition at the solid surface, $v_{\tau}^{s l i p}$ is the slip velocity, defined as the tangential velocity of the fluid at the solid surface measured relative to the (moving) wall, and $L(\phi) \partial_{\tau} \phi$ is the uncompensated Young stress. Physically, $L(\phi)$ measures the deviation from equilibrium at the solid surface. Therefore, equation (2.4) means that the material derivative of $\phi$ at the solid surface is proportional to the deviation of $L(\phi)$ from its equilibrium value zero, and the minus sign ensures this evolution to be relaxational. Equation (2.5) governs the fluid slip at the solid surface. It has been shown that the boundary conditions (2.4) and (2.5) may be derived from Onsager's variational principal as a consistent pair (Qian et al. 2006). Hence we denote them together as the GNBC.

We use $\gamma_{f s}(\phi)=\left(\Delta \gamma_{f s} / 2\right) \sin (\pi \phi / 2)$ which is a smooth interpolation from $\gamma_{f s}\left(\phi_{-}\right)=$ $-\Delta \gamma_{f s} / 2$ to $\gamma_{f s}\left(\phi_{+}\right)=\Delta \gamma_{f s} / 2$. Here $\Delta \gamma_{f s}$ denotes the change of $\gamma_{f s}(\phi)$ from one equilibrium phase of $\phi_{-}=-1$ to the other equilibrium phase of $\phi_{+}=1$, i.e. $\Delta \gamma_{f s}=$ $\gamma_{f s}\left(\phi_{+}\right)-\gamma_{f s}\left(\phi_{-}\right)$. According to the Young equation for the static contact angle $\theta_{s}$, $\gamma_{f s}\left(\phi_{+}\right)+\gamma \cos \theta_{s}=\gamma_{f s}\left(\phi_{-}\right)$, we have $\Delta \gamma_{f s}=-\gamma \cos \theta_{s}$. It can be shown that in the sharp interface limit (Chella \& Vinals 1996; Qian et al. 2003), the uncompensated Young stress satisfies

$$
\int_{\text {int }} \mathrm{d} \tau\left[L(\phi) \partial_{\tau} \phi\right]=\gamma\left(\cos \theta_{d}-\cos \theta_{s}\right)
$$

where $\int_{\text {int }} \mathrm{d} \tau$ denotes the integration across the fluid-fluid interface along the $\tau$ direction and $\theta_{d}$ is the dynamic contact angle.

Note that our choice of $\gamma_{f s}(\phi)$ makes $\partial \gamma_{f s}(\phi) / \partial \phi$ vanish at the two equilibrium phases $\phi_{ \pm}= \pm \sqrt{r / u}= \pm 1$. As a consequence, far away from the contact line and deep in the single-phase region, the phase field becomes a constant in space and time, given by $\phi(\boldsymbol{r}, t)=\phi_{+}$or $\phi(\boldsymbol{r}, t)=\phi_{-}$, which satisfies $\mathrm{d} \phi / \mathrm{d} t \equiv \partial \phi / \partial t+\boldsymbol{v} \cdot \nabla \phi=0$, $\mu=-K \nabla^{2} \phi-r \phi+u \phi^{3}=0$, and $L(\phi)=K \partial_{n} \phi+\partial \gamma_{f s}(\phi) / \partial \phi=0$ at the solid surface. This leads to the disappearance of the transition layer at the solid surface, which in general arises from the competition between the free energies at the surface (represented by $\gamma_{f s}(\phi)$ ) and in the bulk (represented by $F[\phi]$ ). It follows that the 
excess free energy (per unit area of the solid surface) far from the contact line can be simply given by $\gamma_{f s}\left(\phi_{ \pm}\right)$defined at the surface without integrating across the transition layer along the surface normal, and hence the Young equation can be written as $\gamma_{f s}\left(\phi_{+}\right)+\gamma \cos \theta_{s}=\gamma_{f s}\left(\phi_{-}\right)$with the static contact angle $\theta_{s}$ defined at the surface as well.

\subsection{Modelling the immiscible two-phase flows at patterned surfaces}

We consider a binary fluid flowing through a two-dimensional channel in which the top and bottom surfaces are chemically patterned. The binary fluid consists of two immiscible components $\mathrm{A}$ and $\mathrm{B}$. By immiscible components we mean that a (diffuse) interface can be stabilized between them while spatial and temporal variations of the phase field are allowed and governed by the model described in $\$ 2.1$. The top and bottom surfaces are patterned by A-type and B-type stripes, with the A component more attracted by the A-type stripe while the B component is more attracted by the B-type stripe (figure 1). The chemical properties of each stripe are specified by the static contact angle (defined on the side of the B component) and the two slip lengths for the two components. We use $\theta_{s}^{A}$ to denote the static contact angle on the A-type stripe, $l_{s A}^{A}$ and $l_{s B}^{A}$ to denote the slip lengths of the $\mathrm{A}$ and $\mathrm{B}$ components on the A-type stripe, and $\theta_{s}^{B}, l_{s A}^{B}$, and $l_{s B}^{B}$ to denote the corresponding parameters on the B-type stripe. We choose $\theta_{s}^{A}>90^{\circ}, \theta_{s}^{B}<90^{\circ}, l_{s A}^{A}<l_{s B}^{A}$, and $l_{s A}^{B}>l_{s B}^{B}$, considering that a large slip length is realized at a non-wetting fluid-solid interface (e.g. $l_{s B}^{A}$ and $l_{s A}^{B}$ ) (Barrat \& Bocquet 1999). The geometric structure of the pattern is specified by the period of the pattern $\lambda$ and the widths of the A-type and B-type stripes $a$ and $b$ with $\lambda=a+b$. We choose the coordinate system in which the solid surface extends along the $x$-direction, i.e. $\tau=x$, and the outward surface normal $n$ is in the $-z$-direction on the bottom surface and in the $z$-direction on the top surface.

The dimensionless Cahn-Hilliard-Navier-Stokes equations are

$$
\begin{aligned}
& \frac{\partial \phi}{\partial t}+\boldsymbol{v} \cdot \nabla \phi=\mathscr{L}_{d} \nabla^{2}\left(-\nabla^{2} \phi-\phi+\phi^{3}\right), \\
& \mathscr{R}\left[\frac{\partial \boldsymbol{v}}{\partial t}+(\boldsymbol{v} \cdot \nabla) \boldsymbol{v}\right]=-\nabla p+\nabla^{2} \boldsymbol{v}+\mathscr{B}\left(-\nabla^{2} \phi-\phi+\phi^{3}\right) \nabla \phi .
\end{aligned}
$$

The dimensionless GNBC boundary conditions are

$$
\frac{\partial \phi}{\partial t}+v_{x} \partial_{x} \phi=-\mathscr{V}_{s}\left[\partial_{n} \phi-\frac{\sqrt{2}}{3} \cos \theta_{s} s_{\gamma}(\phi)\right]
$$

for the relaxation of $\phi$ at the fluid-solid interface, and the friction law

$$
\left[\mathscr{L}_{s}(\phi)\right]^{-1} v_{x}^{s l i p}=\mathscr{B}\left[\partial_{n} \phi-\frac{\sqrt{2}}{3} \cos \theta_{s} s_{\gamma}(\phi)\right] \partial_{x} \phi-\partial_{n} v_{x}
$$

for the fluid slip, with $s_{\gamma}(\phi)=(\pi / 2) \cos (\pi \phi / 2)$ and $\theta_{s}=\theta_{s}^{A}$ or $\theta_{s}^{B}$. Five dimensionless parameters appear in the above equations. They are (i) $\mathscr{L}_{d}=M r / V \xi$, which is the ratio of the diffusion length $M r / V$ to $\xi$, (ii) $\mathscr{R}=\rho V \xi / \eta$, (iii) $\mathscr{B}=r^{2} \xi / u \eta V=$ $3 \gamma / 2 \sqrt{2} \eta V$, which is inversely proportional to the capillary number $C a=\eta V / \gamma$, (iv) $\mathscr{V}_{s}=K \Gamma / V$, which is the ratio of $K \Gamma$ (of velocity dimension) to $V$, and (v) $\mathscr{L}_{s}(\phi)=\eta / \beta(\phi) \xi$, which is the ratio of the slip length $l_{s}(\phi)=\eta / \beta(\phi)$ to $\xi$. Here $\beta(\phi)=(1-\phi) \beta_{1} / 2+(1+\phi) \beta_{2} / 2$ is the weight-averaged slip coefficient, with $\beta_{1}=\eta / l_{s A}^{A}$ and $\beta_{2}=\eta / l_{s B}^{A}$ on the A-type stripe or $\beta_{1}=\eta / l_{s A}^{B}$ and $\beta_{2}=\eta / l_{s B}^{B}$ on the 


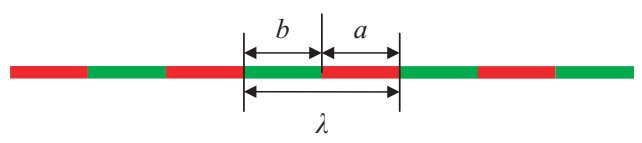

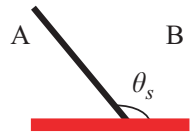

A-type

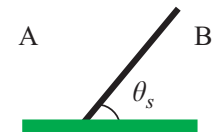

B-type

FIGURE 1. A schematic diagram showing a patterned surface with alternating stripes of different surface properties, represented by the static contact angle and the two slip lengths.

(a)

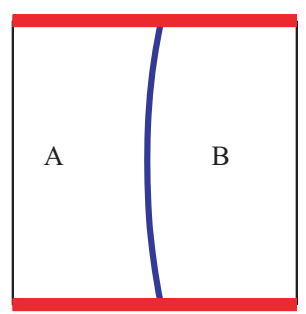

(b)

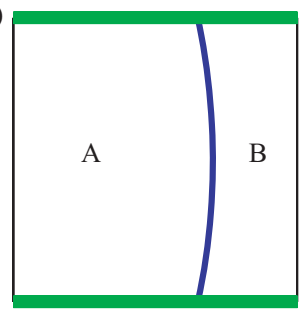

FIGURE 2. The stationary profiles of the dynamic interface (with A fluid on the left and B fluid on the right) in channels with homogeneous surfaces. The flow is from right to left with the averaged velocity of $U=-0.2$. (a) Top and bottom surfaces are both A-type. (b) Top and bottom surfaces are both B-type.

B-type stripe. In our calculations, the values of the first four parameters are taken from those determined through MD simulations (Qian et al. 2003). They are $\mathscr{L}_{d}=5$, $\mathscr{R}=0.03, \mathscr{B}=12$, and $\mathscr{V}_{s}=5$, with the length scale $\xi=0.33 \sigma$ and the velocity scale $V=0.25 \sqrt{\epsilon / m}$ (in which $\epsilon$ and $\sigma$ are the energy and length scales in the Lennard-Jones potential for fluid molecules and $m$ is the fluid molecular mass). We use the above values of $\xi$ and $V$ as the length and velocity units. The dimensionless boundary conditions (2.9) and (2.10) are obtained from equations (2.4) and (2.5), with $\gamma_{f s}(\phi)=\left(\Delta \gamma_{f s} / 2\right) \sin (\pi \phi / 2), \Delta \gamma_{f s}=-\gamma \cos \theta_{s}$, and the dimensionless value $2 \sqrt{2} / 3$ for $\gamma$.

\section{Results and discussion}

We simulate an immiscible two-phase fluid flowing with an average velocity $U$ through a channel with chemically patterned top and bottom surfaces. In most of our simulations, the length of the channel is $L_{x}=100$ and the height of the channel is $L_{z}=40$. The initial conditions for the phase field $\phi$ and the velocity field $v_{x}, v_{z}$ are chosen to be

$$
\begin{aligned}
\phi(x, z, 0) & =\tanh \left(\left(x-x_{0}\right) / \sqrt{2}\right) \\
v_{x}(x, z, 0) & =\frac{6 U}{6 l_{s} / L_{z}+1}\left(l_{s} / L_{z}+z / L_{z}-\left(z / L_{z}\right)^{2}\right) \\
v_{z}(x, z, 0) & =0
\end{aligned}
$$

where $x_{0}$ is the initial interface position. The initial velocity field is a Poiseuille-type quadratic profile in $z$, with an average velocity $U$, which is consistent with the slip boundary condition at the top and bottom boundaries. The boundary conditions (2.4) 
and (2.5) are applied on the top and bottom solid surfaces. At the left and right boundaries, the velocity fields are specified as the above quadratic profiles and $\phi$ is fixed to be -1 or 1 , compatible with the fact that far away from the contact line and deep in the single-phase region, the phase field becomes a constant in space and time, given by $\phi(\boldsymbol{r}, t)=-1$ or $\phi(\boldsymbol{r}, t)=1$. During the simulations, the position of the interface is determined by the zero level set of the function $\phi$. We use a pressure-Poisson solver for the Navier-Stokes equation and a semi-implicit scheme for the Cahn-Hilliard equation. The details of the numerical scheme can be found in Qian et al. (2003).

\subsection{Stick-slip motion and capillary waves}

For homogeneous surfaces, the moving interface can maintain a stationary shape with a dynamical contact angle slightly deviating from the static contact angle $\theta$. Figure $2(a)$ shows the stationary dynamic interface profile in a channel with homogeneous A-type surfaces and figure $2(b)$ shows that in a channel with homogeneous B-type surfaces. In all of the simulations, the A fluid is always on the left (and B fluid on the right) of the interface. When the solid surfaces are patterned, the moving interface has to switch, completely or incompletely, between the two different interface profiles for different homogeneous surfaces, leading to a stick-slip motion of the contact line. We plot two time sequences for the interface profile and contact point velocity in figure 3 where we use $\theta_{s}^{A}=102.4^{\circ}, l_{s A}^{A}=3.8, l_{s B}^{A}=10$ for the A-type stripe and $\theta_{s}^{B}=180^{\circ}-\theta_{s}^{A}=77.6^{\circ}$, $l_{s A}^{B}=10, l_{s B}^{B}=3.8$ for the B-type stripe. Note that the condition $\theta_{s}^{A}+\theta_{s}^{B}=180^{\circ}$ is not necessary since $\theta_{s}^{A}$ and $\theta_{s}^{B}$ are considered independent. Rather, it represents a convenient choice of parameter values.

In the first case (figure $3 a$ ), the interface starts from the A-type (red) region and moves to the left with the average velocity $U=-0.2$. As the interface reaches the B-type (green) region, its profile is rendered energetically unfavourable by the new stripe and the capillary force takes over in adjusting the interface profile. The contact line undergoes an acceleration followed by an deceleration, quickly slipping to the B-type stripe. The maximum slip velocity is about -0.81 which is four times the magnitude of $U$. In the B-type region the interface continues to move with the steady velocity $U=-0.2$ until it reaches the next A-type (red) region. The contact line then slows down (with minimum velocity -0.06), accompanying the adjustment of the interface profile due to the switch of the contact angle. After completing the profile adjustment, the interface continues to move with the steady velocity $U=-0.2$. In the second case (figure $3 b$ ), the surface pattern period is decreased. The above process is seen to repeat as the interface moves through the channel. Figure 4 shows the motion of the interface in the co-moving frame (with a constant velocity $U=-0.2$ ) where the contact line oscillates around a vertical line.

\subsection{Interface breaking and minimum energy dissipation}

We now increase the wettability contrast $r$ defined as the ratio of the static contact angles of the A-type and B-type surfaces, i.e. $r=\theta_{s}^{A} / \theta_{s}^{B}$, and study how the increased contrast affects the interface motion. We choose $\theta_{s}^{A}=152.4^{\circ}$ for the A-type stripe and $\theta_{s}^{B}=27.6^{\circ}$ for the B-type stripe. The values for the four slip lengths remain the same as before. We observe a strong deformation of the interface when the contact line crosses the boundaries between different stripes. This leads to the interesting phenomena of interface breaking and contact line jumping as shown in figure 5 . An enlarged view in figure 6 shows the interface immediately before and after its 
(a)

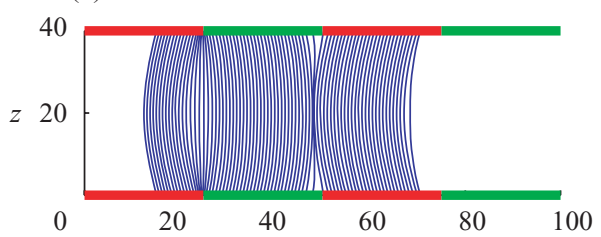

(c)

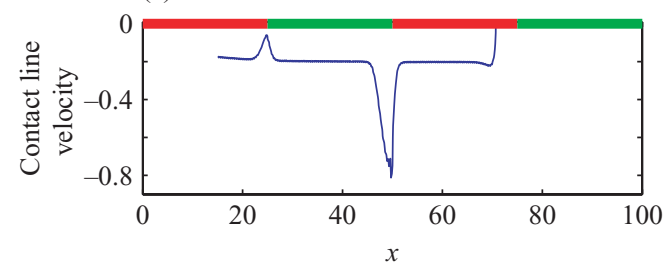

(b)

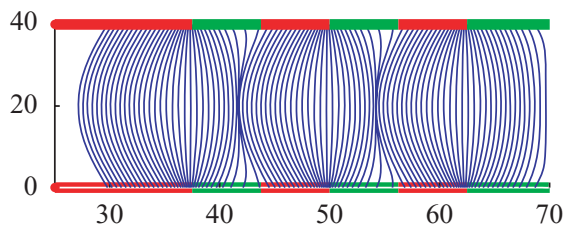

(d)

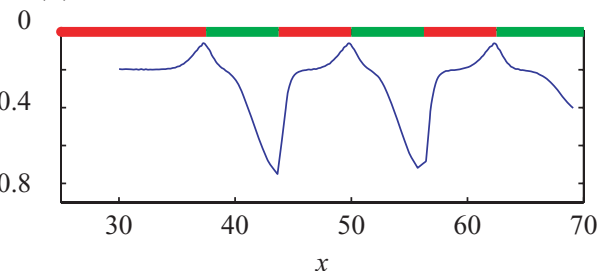

FIGURE 3. Two time sequences, with different surface pattern period, for the interface profile and contact point velocity. The fluid is moving from right to left in each case. The fluid on the left is the A component and that on the right is the B component. As the interface moves away from the domain of the A-type stripe and enters into the domain of the B-type stripe, the contact line velocity increases rapidly, in conjunction with the adjustment of the interface profile. The opposite occurs as the interface crosses the stripe boundary from the B-type stripe to the A-type stripe, and the contact line velocity decreases to almost zero.
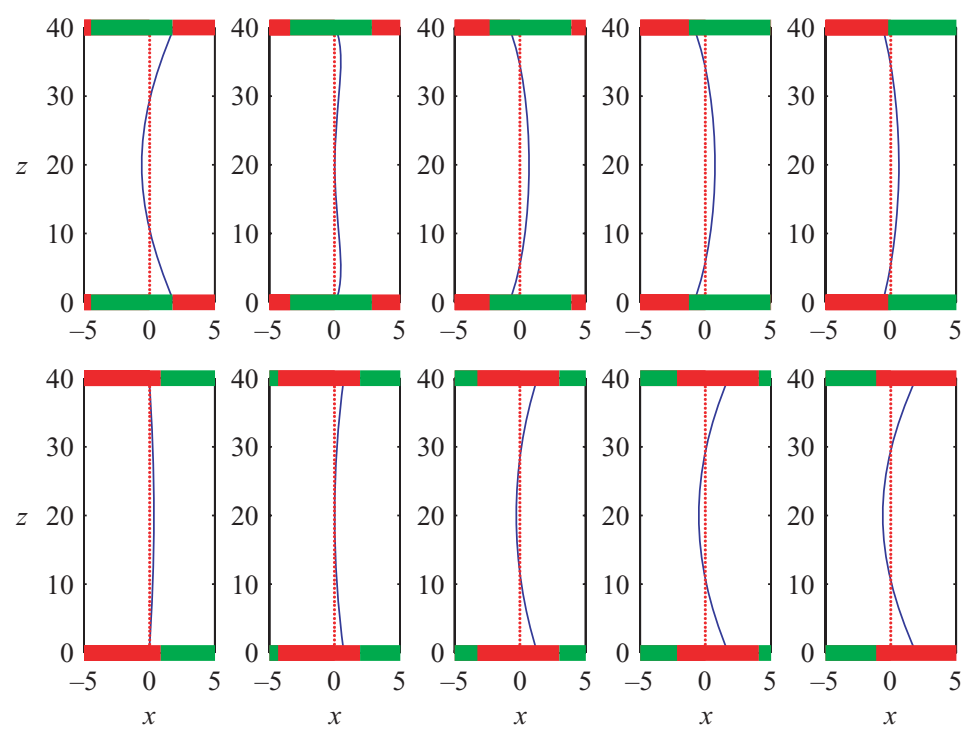

FIGURE 4. Motion of the interface in the co-moving frame, in a time sequence from top left to right, then from bottom left to right. The last frame repeats the first one.

breaking. This is similar to the jump phenomena observed for the moving contact line on undulating surfaces (Luo et al. 2006). The mechanism here is interesting. As seen in figure 6 , breaking of the interface is directly associated with diffusion of the red fluid (the B component of the binary fluid) in space. This diffusion occurs above the A-type stripe which is now strongly non-wetting to the red fluid (with $\theta_{s}^{A}$ close to $180^{\circ}$ ). Unable to fully displace the blue fluid (the A component) that is strongly 
(a)

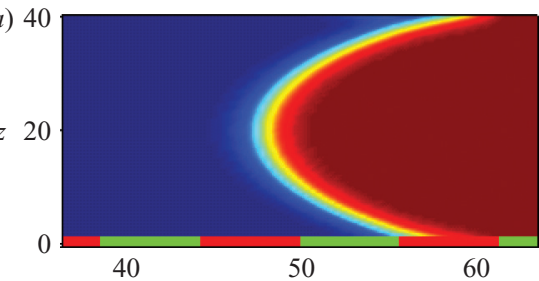

(c)

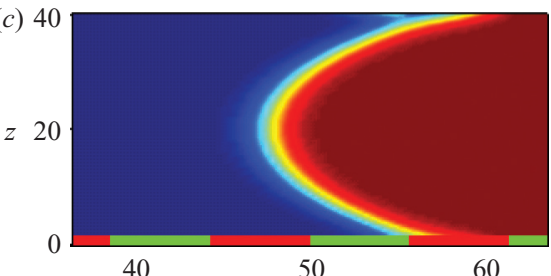

(e)

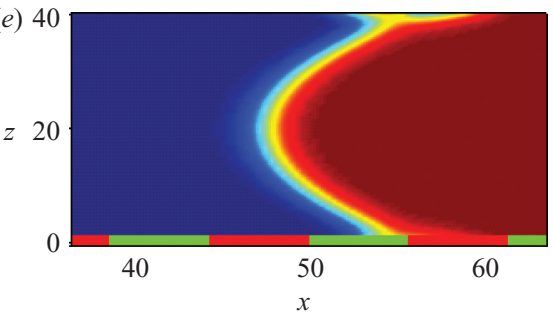

(b) 40

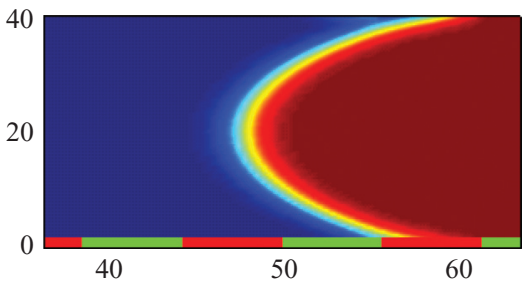

(d) 40

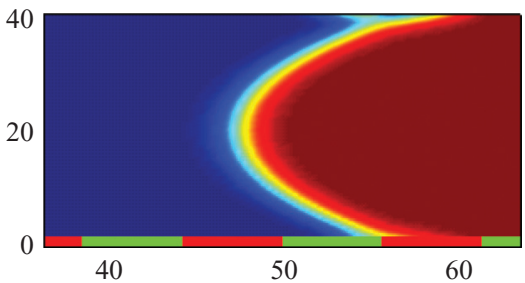

(f) 40

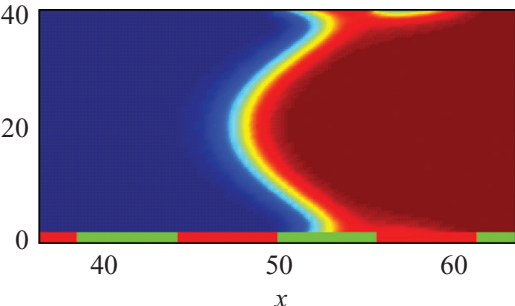

FIGURE 5. Time sequence of an interface moving past a patterned surface with high wettability contrast, as characterized by the two contact angles $\theta_{s}^{A}=152.4^{\circ}$ for the A-type stripe and $\theta_{s}^{B}=27.6^{\circ}$ for the B-type stripe. The break in the interface occurs in the last few frames. An enlarged view is shown in figure 6 . The A component of the binary fluid is coloured blue and the B component is coloured red, to facilitate the visual contrast as well as the diffuse interface between the two.
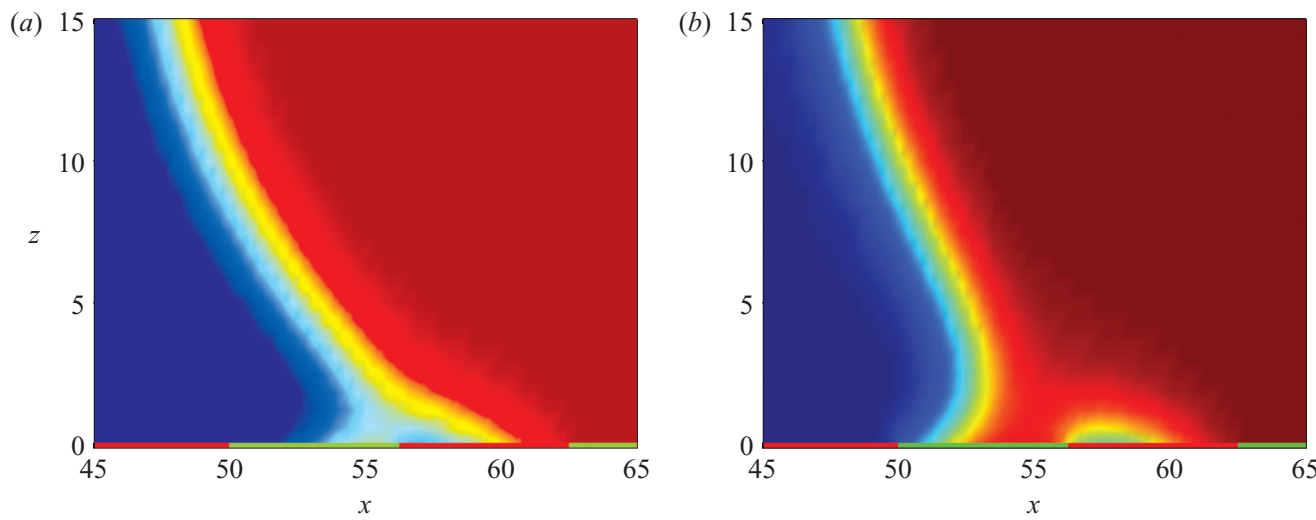

FiguRE 6. An enlarged view of the interface immediately before and after breaking. The red fluid (the B component) on the right prefers the green section (the B-type stripe) of the patterned surface, while the blue fluid (the A component) on the left is strongly attracted to the red section (the A-type stripe). Hence to minimize the total energy dissipation the red fluid avoids the red section of the patterned surface by diffusing across and over the high-energy section. 
attracted to the A-type stripe, the red fluid thus penetrates through the blue fluid via diffusion, and seems to 'grow' out of nowhere onto the next wetting region (the B-type stripe). Visually it thus appears as if the contact line tunnels, or jumps.

The phenomena of interface breaking can be understood from the principle of minimum energy dissipation, the statistical mechanical basis of the GNBC. The total rate of energy dissipation $R_{\text {total }}$ for immiscible two-phase fluid flowing past the patterned surfaces consists of four parts, contributed by the four physically distinct sources of dissipation the shear viscosity in the bulk $R_{v}$, the fluid slip at the solid surface $R_{s}$, the phase field diffusion in the bulk $R_{d}$, and the phase field relaxation at the solid surface $R_{r}$ :

$$
\begin{aligned}
R_{\text {total }} & =R_{v}+R_{s}+R_{d}+R_{r} \\
& =\int \mathrm{d} \boldsymbol{r}\left[\frac{\eta}{2}\left(\partial_{i} v_{j}+\partial_{j} v_{i}\right)^{2}\right]+\int \mathrm{d} S\left[\beta\left(v_{\tau}^{s l i p}\right)^{2}\right]+\int \mathrm{d} \boldsymbol{r}\left[\frac{\boldsymbol{J}^{2}}{M}\right]+\int \mathrm{d} S\left[\frac{\dot{\phi}^{2}}{\Gamma}\right],
\end{aligned}
$$

where $\boldsymbol{J}=-\nabla \mu$ is the diffusive current in the bulk and $\dot{\phi}$ is the material time derivative of $\phi$ at the solid surface.

We calculate the time-averaged dissipation rates over a period with increased wettability contrast $r$. Figure 7 is a plot of time averaged dissipation rates as a function of increasing $r$ from $r=2$ to about 5.5 (as we vary $\theta_{s}^{B}$ from $60^{\circ}$ to $27.6^{\circ}$ and $\theta_{s}^{A}$ from $120^{\circ}$ to $152.4^{\circ}$ ). It is clear that there is a rapid increase of energy dissipation as the contrast ratio $r$ increases but before a critical value $r_{c}$ (close to 3 ) is reached. This rapid increase arises from the complete displacement of the blue fluid that is more and more attracted to the A-type stripe and thus confined in a wedge of smaller and smaller angle. The critical value $r_{c}$ corresponds to the onset of interface breaking. Above the critical value, the system finds an alternative channel to reduce the energy dissipation which would otherwise further increase. This is achieved by the interface breaking. As one can see from figure 7, for $r<r_{c}$ (before interface breaking), the dissipation due to slip $R_{s}$ is much large than that due to diffusion $R_{d}$. For $r>r_{c}, R_{s}$ decreases rapidly while $R_{d}$ continues to increase, indicating strong diffusion.

\subsection{Scaling for the energy dissipation}

When immiscible fluid flows over a homogeneous surface with a velocity $U$, the motion is steady and the rate of energy dissipation scales as $R \propto U^{2}$ for small $U$, following the general rule governing the entropy production in a thermodynamic process (essentially a Taylor expansion in $U$ for positive definite $R$, see Landau \& Lifshitz 1997). On patterned surfaces, the contact line movement shows a stick-slip behaviour and the interface profile oscillates in time, thus inducing extra dissipation. We are interested in how the dissipation scales with the average velocity $U$ for this oscillatory case. The results in the previous section have indicated that the rate of energy dissipation is also affected by the surface wettability contrast which leads to two different energy dissipation regimes separated by a critical wettability contrast ratio $r_{c}$. We examine the capillary-wave-induced extra dissipation (Zhou \& Sheng 1990) before the interface breaking. We calculate the rates of dissipation $R_{v}, R_{s}, R_{d}$, $R_{r}$ and the total dissipation $R_{\text {total }}$ for $U=-0.05, \theta_{s}^{A}=102.4^{\circ}$, and $\theta_{s}^{B}=77.6^{\circ}$. Figure 8 shows that each of the four components of dissipation remains a constant when the interface moves steadily in either the A-type region or the B-type region. As the contact line moves across the boundary from the A-type to the B-type stripe, it undergoes a fast slip due to the change in the surface property and there is a significant jump in $R_{\text {total }}$, dominated by the contributions from $R_{v}$ and $R_{s}$. This is 


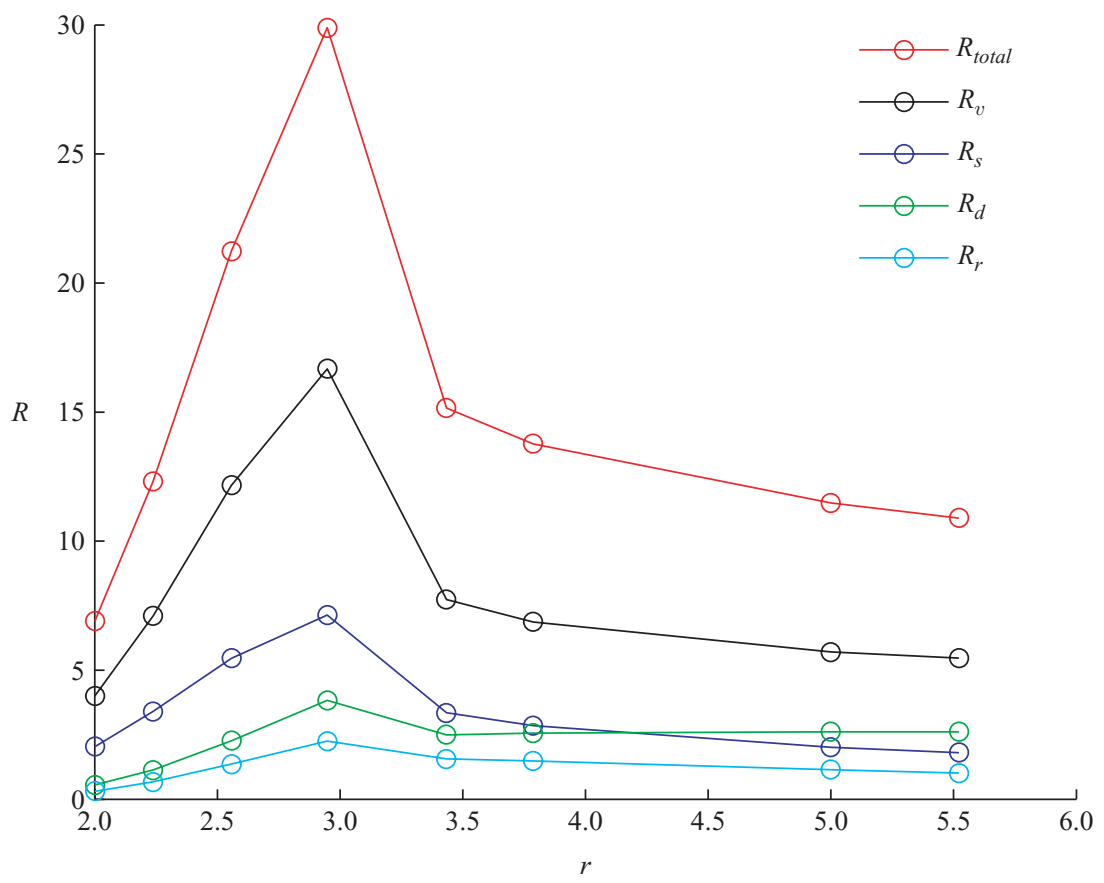

FIGURE 7. Time-averaged dissipation rates $R$ vs. the wettability contrast $r$.

physically understandable because a jump in $R_{v}$ is generated by the capillary-wave excitation at the fluid-fluid interface while a jump in $R_{s}$ is generated by the rapid slipping of the contact line.

To study the behaviour of the capillary-wave-induced extra dissipation, we subtract the usual steady motion dissipation on a uniform surface (which scales as $U^{2}$ ) and examine the scaling behaviour of the extra dissipation through time averaging. Our results show that the rate of extra energy dissipation has certain universal behaviour when the contrast ratio $r$ is close to the critical value $r_{c}$. Figure 9 shows the time sequences (maximum shifted to $t=0$ ) of the extra dissipation $R_{v}$ (viscous dissipation) and $R_{s}$ (due to slip) for three different speeds $U=-0.01,-0.0075$ and -0.005 on a surface with contrast ratio $r=2.6$. The three profiles match almost perfectly, showing that the capillary-wave-induced extra dissipation depends on the surface pattern only. Physically, the capillary wave excitation arises from the change in the static wetting property (contact angle) over the patterned surface and becomes independent of the displacement speed when it approaches zero. The time-averaged dissipation over a time period $T$ (corresponding to the interface motion past one period of the surface pattern $\lambda$ ) gives

$$
\frac{1}{T} \int_{0}^{T} R(t) \mathrm{d} t=\left(\frac{1}{\lambda} \int_{0}^{T} R(t) \mathrm{d} t\right) \frac{\lambda}{T}=c|U|,
$$

where $c=1 / \lambda \int_{0}^{T} R(t) \mathrm{d} t$. Since $R(t)$ decays very fast to zero away from the maximum and is independent of $U, c$ is therefore independent of $U$. Thus, the time-averaged dissipation rates scale as $R_{v}, R_{s} \propto|U|$.

We have also verified this linear $U$ scaling relationship numerically for wettability contrast $r=2.6$. The time-averaged total rate of dissipation and all four components are log-log plotted as functions of $U$ in figure 10. It is shown that $R_{d}$, the rate of 

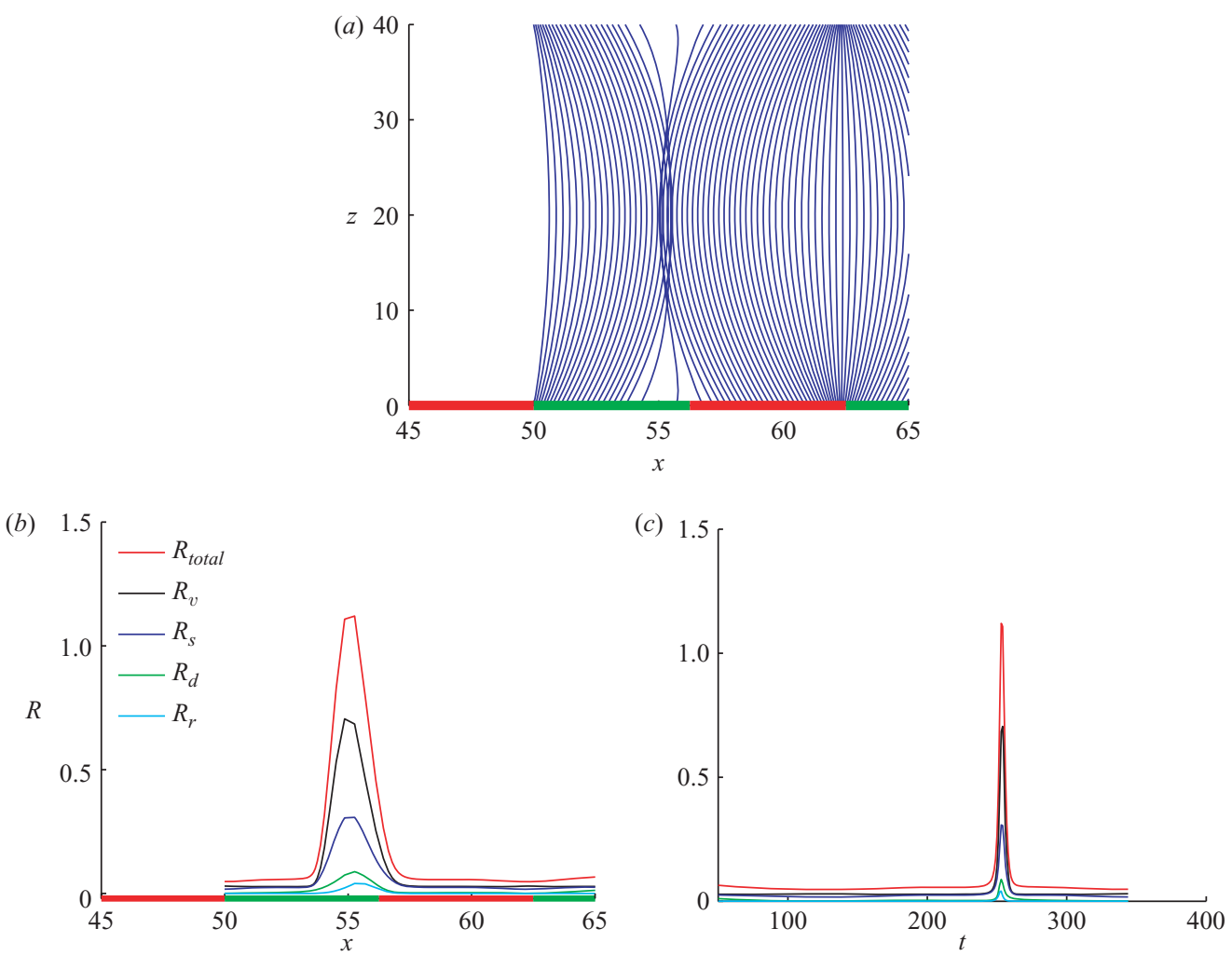

FIgURE 8. (a) The interface profiles during the contact line slip across the boundary from the A-type to the B-type stripe, and the corresponding rates of energy dissipation plotted as function of the position of the contact point $(b)$ and plotted as function of time $(c)$.

dissipation due to diffusion, remains almost constant. However, the viscous dissipation rate $R_{v}$ and that due to slip $R_{s}$ behave differently when $U$ is close to zero. A numerical estimation of the slopes confirms the scaling relation

$$
R_{v}, R_{s} \propto|U|
$$

in the limit of small $U$. This behaviour arises from the inhomogeneity of the dissipation in time. As $U$ approaches zero, the dissipation is dominated by the A-to-B switch associated with the fast contact line slip across the boundary from the A-type to the B-type stripe. Averaging over a time period $T$ then results in a dissipation rate proportional to $U$, as expressed in equation (3.1). At intermediate contrast ratios $1 \leqslant r<r_{c}$, the moving contact line dissipation would appear to scale as $U^{\alpha}$, with $1<\alpha \leqslant 2$.

\subsection{Effective properties of the patterned surface}

As the period of the pattern $\lambda$ becomes small enough, the patterned surface acts effectively as a homogeneous surface. This requires the hydrodynamic relaxation of the fluid-fluid interface to be much slower than its translation over the solid surface. As shown in figure 4, the interfacial profile undergoes a periodic capillary-wave excitation in the co-moving frame, induced by the jump in surface properties at the boundary of different stripes. In addition, the time scale associated with this hydrodynamic relaxation of the interface is well defined in the limit of small displacement speed (see 

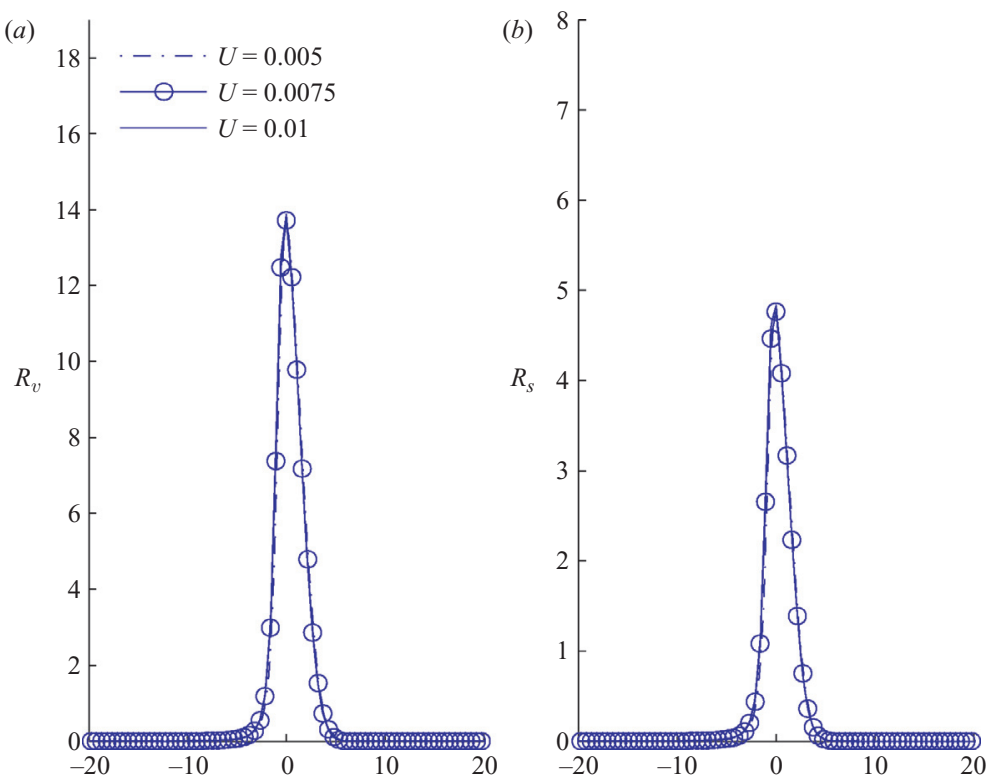

FIGURE 9. The time-shifted extra dissipation $R_{v}(a)$ and $R_{s}(b)$ for three different speeds $U=-0.01, U=-0.0075$ and $U=-0.005$ at contrast $r=2.6$.

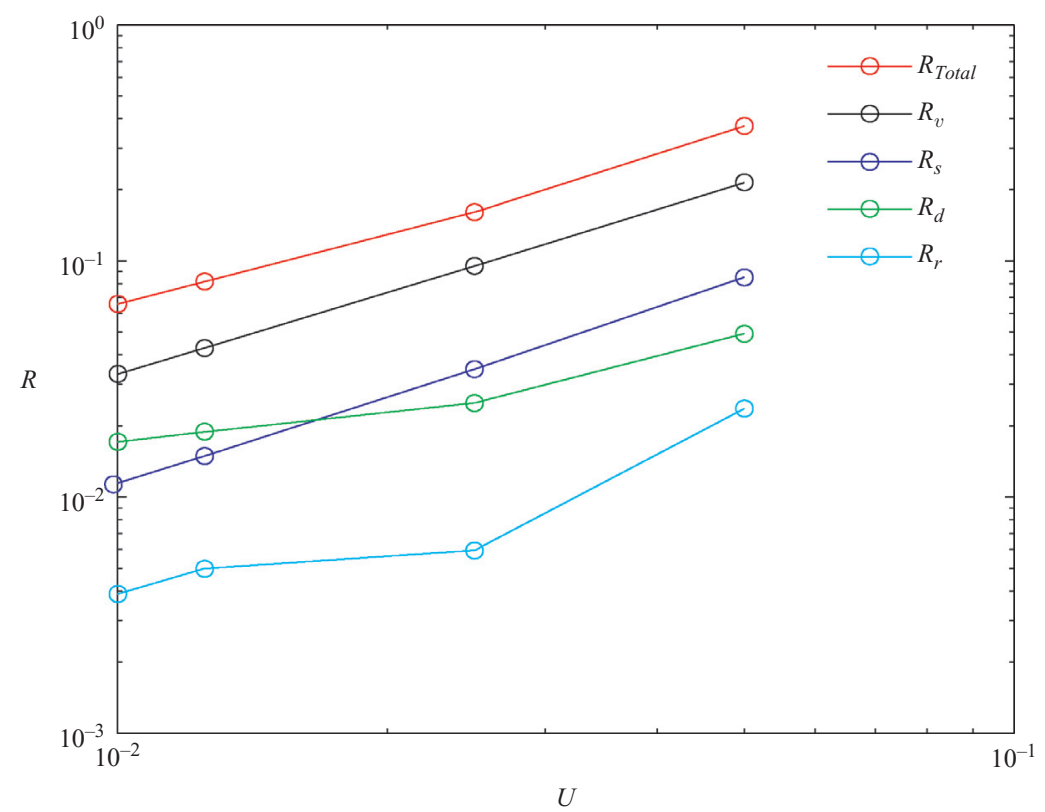

FiguRE 10. Log-log plot of $R$ vs. $U$. Here $R$ represents the total rate of energy dissipation $R_{\text {total }}$ and its four components, $R_{v}, R_{s}, R_{d}$, and $R_{r}$. Numerical estimation confirms the $R \propto U$ scaling.

figure 9). If this time scale becomes much longer than the time period $T=\lambda /|U|$, then the interfacial profile can undergo very little change in each period and the capillary-wave excitation is suppressed. That is, the interface is not able to respond to the jump in surface properties. Instead, it keeps a nearly constant profile and 
moves at a nearly constant speed, as if over a homogeneous surface. It is interesting to study the effective properties of the patterned surface in this regime, and to see how they depend on the ratio $a / b$ of the respective widths of the A-type and B-type stripes. Figure 11 shows the simulation results obtained for a set of small periods of the pattern $(\lambda=6.25,3.125,1.5625)$ with the fixed ratio $a / b=1$. When the period of the pattern is small enough, the interface can never fully relax to the stationary profiles in the A-type and B-type regions. While the velocity of the contact line still oscillates around the steady velocity $U=-0.2$, the oscillation amplitude decreases with the decreasing period. In the case of $\lambda=1.5625$, the moving interface is almost vertical to the solid surfaces, with an effective dynamic contact angle close to $90^{\circ}$. The effective properties of the patterned surface can be tuned as well by the ratio $a / b$. As shown in figure 12 , when $a / b$ is changed to $1 / 3$, we have a moving interface with a contact angle closer to that of the stationary interface in the B-type region (when it is sufficiently wide). Similarly, for $a / b=3$, we observe a moving interface closer to the stationary one in the A-type region (when it is sufficiently wide). This suggests a way to design and control the dynamic wetting properties of the surface by tuning the period $\lambda$ and the ratio $a / b$. The numerical results suggest that the effective contact angle $\theta_{e}$ is approximately given by $\cos \theta_{e} \approx \alpha \cos \theta_{s}^{A}+(1-\alpha) \cos \theta_{s}^{B}$ where $\alpha=a / \lambda$.

For a patterned surface that effectively acts as a homogeneous surface, one can derive the effective contact angle $\theta_{e}$ by a simple argument. For this purpose, we use $L(\phi)=K \partial_{n} \phi+\partial \gamma_{f s}(\phi) / \partial \phi$ defined at the solid surface, in which the contact angle and the change of the fluid-solid interfacial free energy across the fluid-fluid interface are both included. For gently curved interfaces, multiplying $K \partial_{n} \phi$ by $\partial_{x} \phi$ and integrating across the fluid-fluid interface along $x$ yields

$$
\int_{i n t} \mathrm{~d} x\left(K \partial_{n} \phi\right) \partial_{x} \phi=\int_{i n t} \mathrm{~d} \phi\left(K \partial_{m} \phi\right) \cos \theta
$$

where $\int_{i n t} \mathrm{~d} x$ denotes integration across the fluid-fluid interface along $x$, and $\partial_{m}$ means taking spatial derivative along the fluid-fluid interface normal $m$, with $\partial_{n} \phi$ approximated by $\partial_{m} \phi \cos \theta$. Here $\theta$ is the contact angle. From $\int_{i n t} \mathrm{~d} \phi\left(K \partial_{m} \phi\right)=$ $\int_{i n t} \mathrm{~d} m K\left(\partial_{m} \phi\right)^{2}=\gamma$, we obtain $\int_{i n t} \mathrm{~d} x\left(K \partial_{n} \phi\right) \partial_{x} \phi=\gamma \cos \theta$. Multiplying $\partial \gamma_{f s}(\phi) / \partial \phi$ by $\partial_{x} \phi$ and integrating across the fluid-fluid interface along $x$ yields

$$
\int_{i n t} \mathrm{~d} x\left[\partial \gamma_{f s}(\phi) / \partial \phi\right] \partial_{x} \phi=\int_{i n t} \mathrm{~d} \phi\left[\partial \gamma_{f s}(\phi) / \partial \phi\right],
$$

which equals $\gamma_{f s}\left(\phi_{+}\right)-\gamma_{f s}\left(\phi_{-}\right)=\Delta \gamma_{f s}$, the change of the fluid-solid interfacial free energy across the fluid-fluid interface. From the above relations, we obtain

$$
\int_{i n t} \mathrm{~d} x\left[L(\phi) \partial_{x} \phi\right]=\int_{i n t} \mathrm{~d} x\left[K \partial_{n} \phi+\frac{\partial \gamma_{f s}(\phi)}{\partial \phi}\right] \partial_{x} \phi=\gamma \cos \theta+\Delta \gamma_{f s},
$$

which reduces to the Young equation $\gamma \cos \theta_{s}+\Delta \gamma_{f s}=0$ for $L(\phi)=0$ in equilibrium, with the static contact angle $\theta_{s}$ equalling $\theta_{s}^{A}$ and $\theta_{s}^{B}$ on the A-type and B-type stripes, respectively.

The value of the integral $\int_{i n t} \mathrm{~d} x\left[\partial \gamma_{f s}(\phi) / \partial \phi\right] \partial_{x} \phi=\Delta \gamma_{f s}$ alternates between $-\gamma \cos \theta_{s}^{A}$ and $-\gamma \cos \theta_{s}^{B}$ as the interface moves over the patterned surface. For sufficiently small period $\lambda$, the interface moves with a nearly constant velocity. This is seen on the right panels of figure 11, where the velocity fluctuations are seen to decrease in magnitude as $\lambda$ decreases. Therefore, taking the time average of $\Delta \gamma_{f s}$ is equivalent to averaging it over the position of the interface (or contact line) along the 

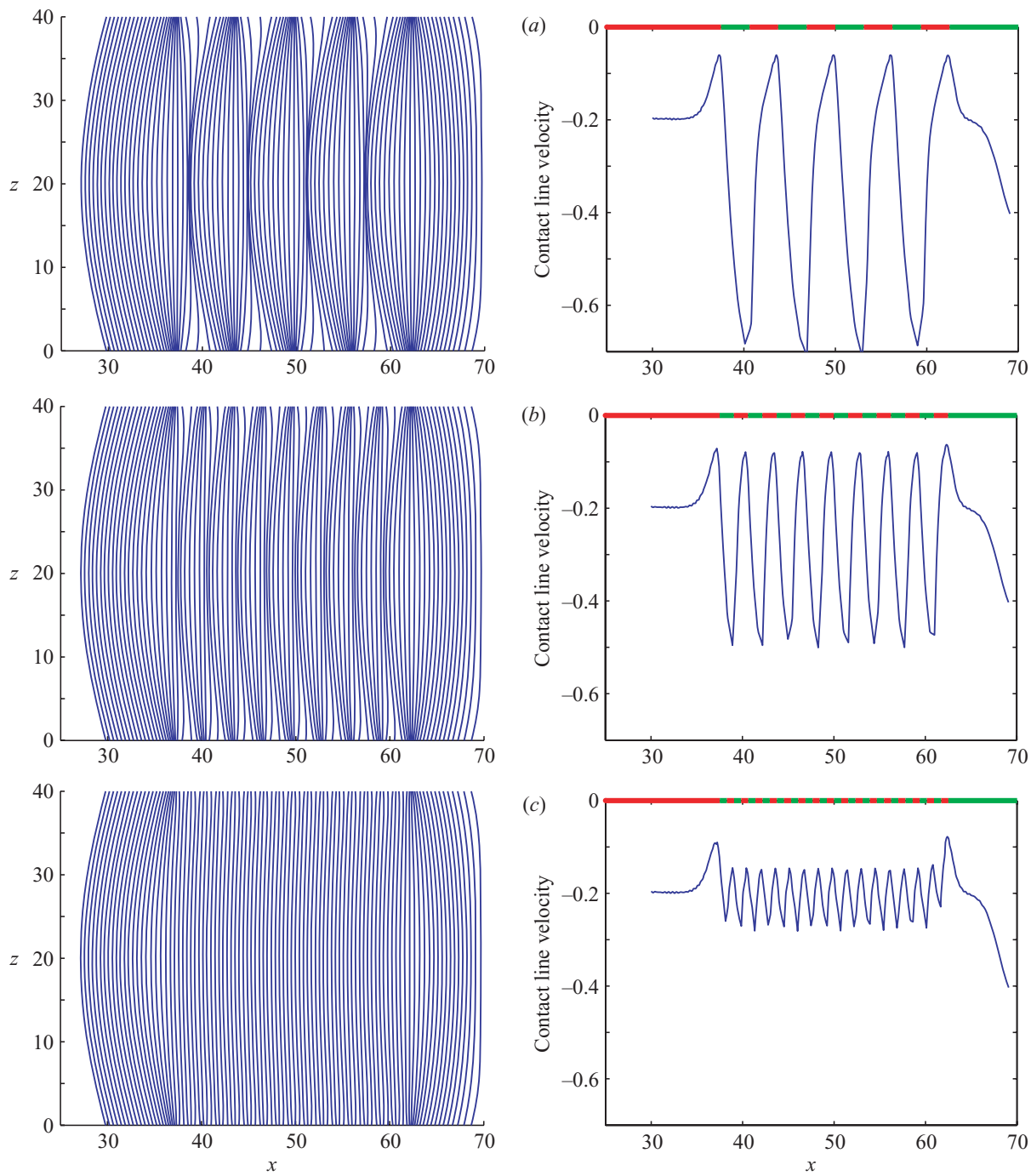

FIGURE 11. The interface profiles and contact-line velocities obtained for a set of decreasing periods of the pattern: $\lambda=6.25(a), 3.125(b)$, and $1.5625(c)$.
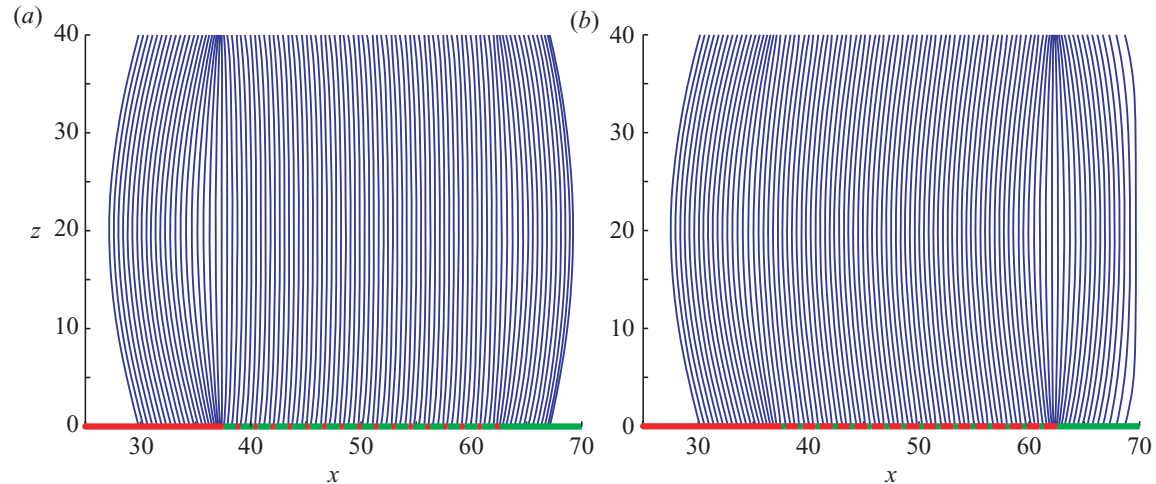

FIGURE 12. The interface profiles for $a / b=1 / 3(a)$ and $a / b=3(b)$. The effective contact angle of the patterned surface is tuned by the ratio $a / b$. 
(a)

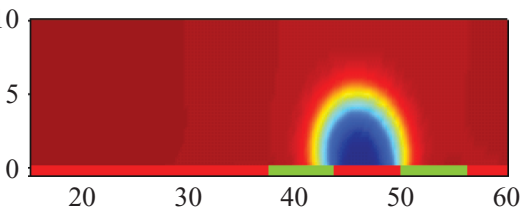

(c) 10

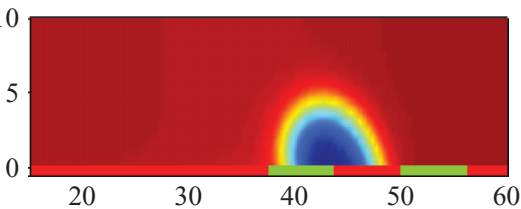

(e)

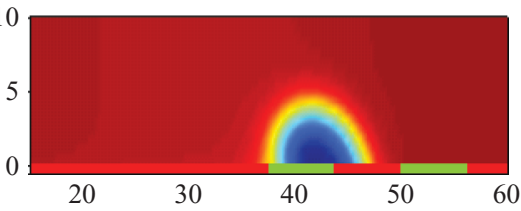

(g)

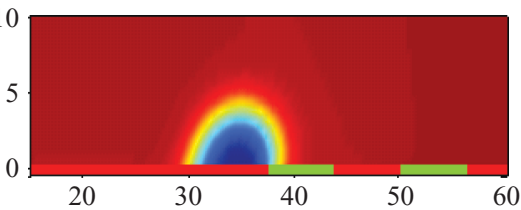

(b) 1

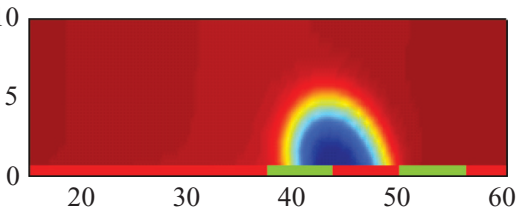

(d) 10

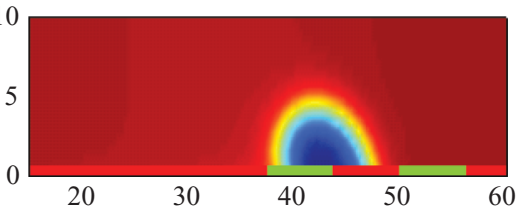

(f) 10

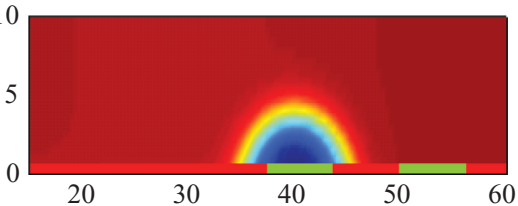

(h) 1

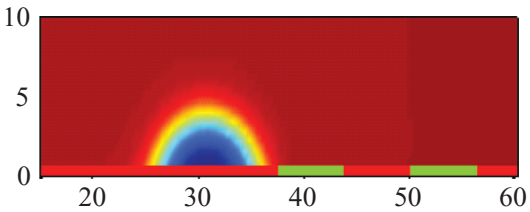

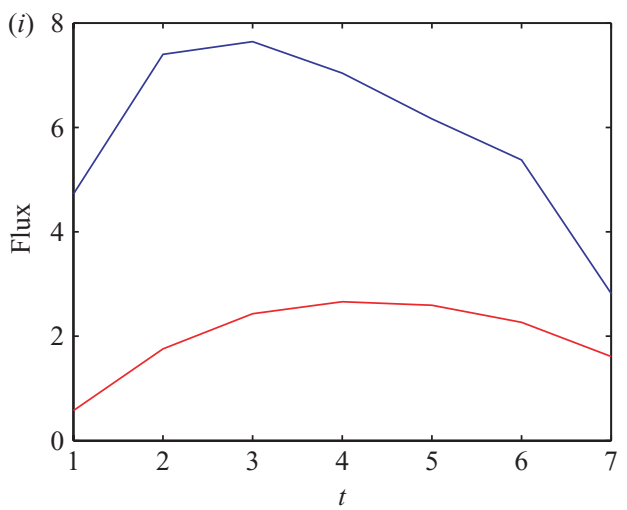

FIGURE 13. $(a-h)$ Equally spaced time sequence plot of a droplet (with initial diameter 8 ) of the blue fluid (the A component). The wettability contrast for the droplet fluid over the A-type and B-type stripes of the patterned surface is low. (i) The time variations of the convection flux (blue line) and the negative of the diffusion flux (red line) at the boundary of the B-type stripe crossed. Convection clearly dominates.

surface, which is given by

$$
\frac{1}{\lambda}\left[a\left(-\gamma \cos \theta_{s}^{A}\right)+b\left(-\gamma \cos \theta_{s}^{B}\right)\right]=-\gamma\left[\alpha \cos \theta_{s}^{A}+(1-\alpha) \cos \theta_{s}^{B}\right] .
$$

Meanwhile, since the shape of the interface changes very little in time, the effective contact angle $\theta_{e}$ can be obtained from $\int_{i n t} \mathrm{~d} x\left(K \partial_{n} \phi\right) \partial_{x} \phi=\gamma \cos \theta_{e}$, which is nearly constant because the phase field $\phi$ is translated by a nearly constant velocity. For slow displacement, the time average of $\int_{i n t} \mathrm{~d} x\left[L(\phi) \partial_{x} \phi\right]$ should approach zero. That 
(a)

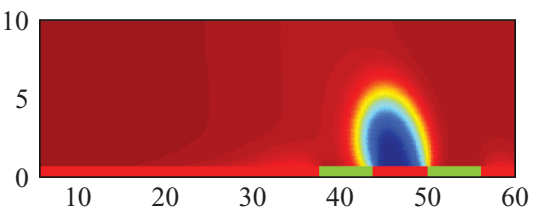

(c)

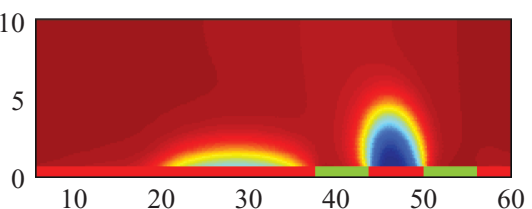

(e)

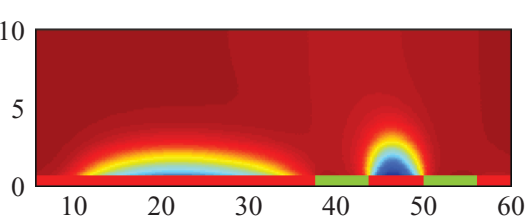

$(g)$

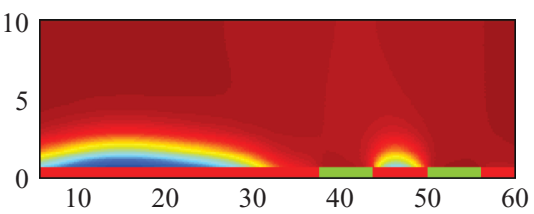

(b)

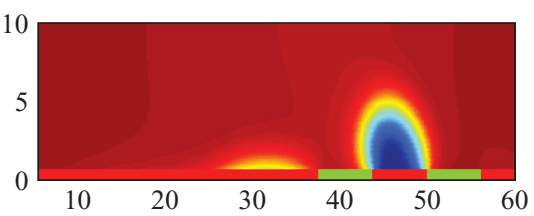

(d)

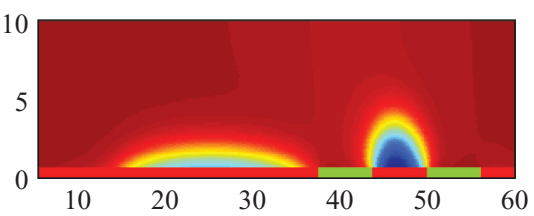

(f)

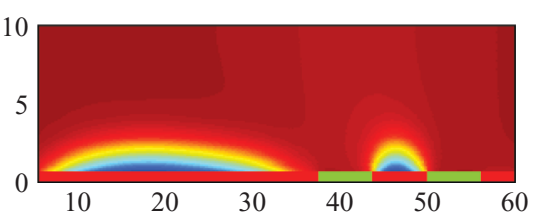

(h)

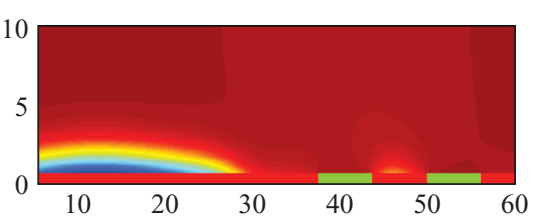

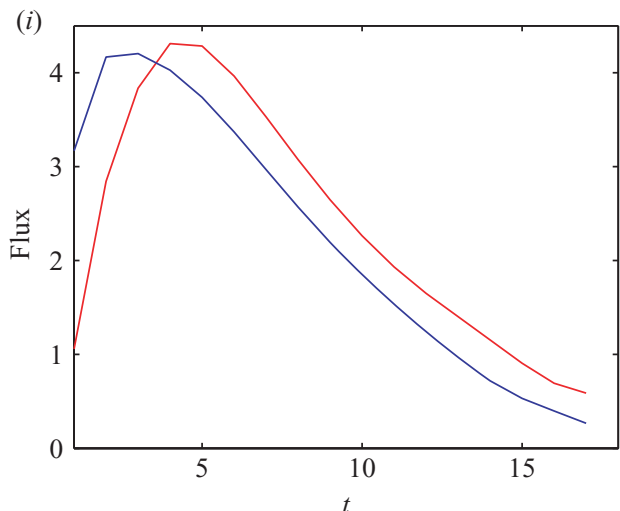

Figure 14. Same as figure 13 , but plotted for a high wettability contrast with $\theta_{s}^{A}=152.4^{\circ}$ at the A-type stripes and $\theta_{s}^{B}=27.6^{\circ}$ at the B-type stripes. Compared to figure 13, a qualitatively different behaviour is obtained, as the diffusion is now responsible for most of the droplet fluid (the A component) transport across the high energy part (the B-type green section) of the patterned surface. In contrast to that in figure 13, in $(i)$ now diffusion flux carries more of the droplet fluid across the high-energy section of the surface.

is, the sum of $\gamma \cos \theta_{e}$ and the time average of $\Delta \gamma_{f s}$ in equation (3.3) should approach zero, i.e.

$$
\gamma \cos \theta_{e}-\gamma\left[\alpha \cos \theta_{s}^{A}+(1-\alpha) \cos \theta_{s}^{B}\right] \rightarrow 0
$$

which yields

$$
\cos \theta_{e} \rightarrow \alpha \cos \theta_{s}^{A}+(1-\alpha) \cos \theta_{s}^{B}
$$


That means the patterned surface acts as a homogeneous surface at which the effective static contact angle $\cos \theta_{s}^{e}$ is given by

$$
\cos \theta_{s}^{e}=\alpha \cos \theta_{s}^{A}+(1-\alpha) \cos \theta_{s}^{B} .
$$

Note that our specific choice of $\gamma_{f s}(\phi)$ leads to the disappearance of a transition layer (of finite thickness $\sim \xi$ ) at the solid surface. As a consequence, the excess free energy per unit area is given by $\gamma_{f s}\left(\phi_{ \pm}\right)$at the surface, and the Young equation takes the simple form of $\gamma \cos \theta_{s}+\Delta \gamma_{f s}=0$. This is still true for the patterned surfaces and results in the effective contact angle $\theta_{s}^{e}$ in equation (3.4).

\subsection{Diffusion-dominated motion of a droplet}

The effects of interface breaking and contact line jumping on an interface moving over a patterned surface are further investigated by varying the relative volume fraction of the two fluid components. Consider a droplet (with diameter 8) of the A component (coloured blue) surrounded by the B component (coloured red). The droplet is initially centred at $x=50$ and is pushed to the left by an external force, moving with the velocity $U=-0.8$. In the case of low contrast of wettability $\left(\theta_{s}^{A}=109.4^{\circ}\right.$ at the A-type stripes of the surface and $\theta_{s}^{B}=70.6^{\circ}$ at the B-type stripes), the droplet can move from one A-type stripe to the next by crossing a B-type stripe in between with only a slight change of shape (figure 13).

However, for significantly increased wettability contrast $\left(\theta_{s}^{A}=152.4^{\circ}\right.$ at the A-type stripes and $\theta_{s}^{B}=27.6^{\circ}$ at the B-type stripes), the droplet of the A component is no longer able to move across the B-type stripe as a whole, because the droplet fluid is strongly repelled by the B-type stripe, and thus is unable to fully displace the surrounding fluid that is strongly attracted to that section of the surface (figure 14). Instead, the droplet fluid is transported across the B-type stripe by diffusion above that part of the patterned surface. A plot of the diffusion and convection fluxes at the boundary of the B-type stripe that is crossed shows that in the low contrast case, convection dominates over diffusion, while in the high contrast case the reverse is true.

\section{Conclusion}

We have presented numerical results of contact line dynamics on chemically patterned surfaces. The simulations are performed based on a diffusive interface model together with the generalized Navier boundary conditions. The numerical results revealed many interesting phenomena of the moving contact line on patterned surfaces. We investigated how the extra dissipation induced by the stick-slip motion of the contact line scales with the driving velocity $U$. The dependence of the effective properties on the geometry of the patterned surfaces suggests a way to design and control the dynamic wetting properties of the surfaces by tuning the period and the ratio of the pattern. Increasing the wettability contrast leads to interface breaking in which diffusion becomes dominant. It is important to note that these phenomena cannot be obtained computationally without the consideration of a diffuse interface in the present model, in which the uncompensated Young stress acts together with the bulk diffusion and surface relaxation of the phase field describing the interface. In particular, a sharp interface description (in which the interface is impenetrable) would yield a very different dynamic behaviour. It has been shown that the present model can be derived from the principle of minimum energy dissipation (Qian et al. 2006), a principle that underlies all linear dissipative responses in irreversible thermodynamic 
processes (Onsager 1931a,b; Onsager \& Machlup 1953). In order to minimize the total rate of energy dissipation, for example, the high wettability contrast case calls for the breaking of the interface via diffusion, a route present in our model but absent if the interface is modelled to be impenetrable. The bulk diffusion is coupled with the surface relaxation and slipping, governed respectively by the boundary conditions (2.9) and (2.10), which have been shown to be the consequence of the minimum energy dissipation principle (Qian et al. 2006).

This work is partially supported by the Hong Kong RGC grant HKUST 604803, the RGC Central Allocation Grant CA05/06.SC01 and the Croucher Foundation Grant Z0138.

\section{REFERENCES}

BARRAT, J.-L. \& Bocquet, L. 1999 Large slip effect at a nonwetting fluid-solid interface. Phys. Rev. Lett. 82, 4671.

Blake, T. D. \& Haynes, J. M. 1969 Kinetics of liquid/liquid displacement. J. Colloid Interface Sci. 30, 421-423.

Briant, A. J. \& Yeomans, J. M. 2004 Lattice Boltzmann simulations of contact line motion. II. Binary fluids. Phys. Rev. E 69, 031603.

CAHn, J. W. \& Hilliard, J. E. 1958 Free energy of a nonuniform system. I. Interfacial free energy. J. Chem. Phys. 28, 258-267.

Chella, R. \& Vinals, J. 1996 Mixing of a two-phase fluid by cavity flow. Phys. Rev. E 53, 3832-3840.

Chen, H. Y., JASNOW, D. \& VinALS, J. 2000 Interface and contact line motion in a two phase fluid under shear flow. Phys. Rev. Lett. 85, 1686-1689.

Cubaud, T. \& Fermigier, M. 2004 Advancing contact lines on chemically patterned surfaces. $J$. Colloid Interface Sci. 269, 171-177.

Darhuber, A. A., Troian, S. M., Miller, S. M. \& Wagner, S. 2000 Morphology of liquid microstructures on chemically patterned surfaces. J. Appl. Phys. 87, 7768.

Dussan V., E. B. \& Davis, S. H. 1974 On the motion of a fluid-fluid interface along a solid surface. J. Fluid Mech. 65, 71-95.

Dussan V., E. B. 1979 On the spreading of liquids on solid surfaces: static and dynamic contact lines. Annu. Rev. Fluid Mech. 11, 371.

De Gennes, P. G. 1985 Wetting: statics and dynamics. Rev. Mod. Phys. 57, 827.

Gau, H., Herminghaus, S., Lenz, P. \& Lipowsky, R. 1999 Liquid morphologies on structured surfaces. Science 283, 46-49.

Hocking, L. M. 1977 A moving fluid interface. Part 2. The removal of the force singularity by a slip flow. J. Fluid Mech. 79, 209-229.

Huh, C. \& Mason, S. G. 1977 The steady movement of a liquid meniscus in a capillary tube. $J$. Fluid Mech. 81, 401-419.

Huh, C. \& Scriven, L. E. 1971 Hydrodynamic model of steady movement of a solid/liquid/fluid contact line. J. Colloid Interface Sci. 35, 85-101.

JACQMIN, D. 2000 Contact-line dynamics of a diffuse fluid interface. J. Fluid Mech. 402, 57-88.

Kusumaatmaja, H., Lopolds, J., Dupuis, A. \& Yeomans, J. M. 2006 Drop dynamics on chemically patterned surfaces. Europhys. Lett. 73, 740-746.

Kuksenok, O., Jasonw, D., Yeomans, J., \& Balazs, A. 2003 Periodic droplet formation in chemically patterned microchannels. Phys. Rev. Lett. 91, 108303.

Landau, L. D. \& Lifshitz, E. M. 1997 Statistical Physics (Part 1). Oxford University Press.

Luo, X, Wang, X. P., Qian, T. Z. \& Sheng, P. 2006 Moving contact line over undulating surfaces. Solid State Commun. 139 623-629.

Onsager, L. 1931 a Reciprocal relations in irreversible processes. I. Phys. Rev. 37, 405-426.

Onsager, L. $1931 b$ Reciprocal relations in irreversible processes. II. Phys. Rev. 38, 2265-2279.

Onsager, L. \& Machlup, S. 1953 Fluctuations and irreversible processes. Phys. Rev. 91, 1505-1512.

Pismen, L. M. \& PomeaU, Y. 2000 Disjoining potential and spreading of thin liquid layers in the diffuse-interface model coupled to hydrodynamics. Phys. Rev. E 62, 2480-2492. 
Qian, T. Z., Wang, X. P. \& Sheng, P. 2003 Molecular scale contact line hydrodynamics of immiscible flows. Phys. Rev. E 68, 016306.

Qian, T. Z., WANG, X. P. \& Sheng, P. 2004 Power-law slip profile of the moving contact line in two-phase immiscible flows. Phys. Rev. Lett. 93, 094501.

Qian, T. Z., WANG, X. P. \& Sheng, P. 2006 A variational approach to the moving contact line hydrodynamics, J. Fluid Mech. 564, 333-360.

SePpecher, P. 1996 Moving contact lines in the Cahn-Hilliard theory. Intl J. Engng Sci. 34, 977-992.

Zhou, M. Y. \& Sheng, P. 1990 Dynamics of immiscible-fluid displacement in a capillary tube. Phys. Rev. Lett. 64, 882-885. 